\title{
Immobilized culture of nonadherent cells on an oleyl poly(ethylene glycol) ether-modified surface
}

\author{
Koichi Kato ${ }^{1}$, Kohei Umezawa ${ }^{2}$, Daniel P. Funeriu ${ }^{1}$, Masato Miyake$^{1}$, \\ Jun Miyake ${ }^{1}$, and Teruyuki Nagamune ${ }^{2}$
}

BioTechniques 35:1014-1021 (November 2003)

\begin{abstract}
Microarrays of living cells are an emerging tool in systems such as reverse transfection. These studies are limited to adherent cells partly because of the difficulty of cell immobilization. Using a newly developed reagent, the biocompatible anchor for membrane (BAM), we show herein the rapid and strong attachment of living nonadherent cells and adherent cells on BAM-modified surfaces. Normal cellular growth was observed for over 7 days on BAM-modified surfaces. We expect this methodology to greatly expand the scope of current cell microarray technology.
\end{abstract}

\section{INTRODUCTION}

The construction of patterned cell cultures on chemically modified solid supports is of particular importance in cell-based microarrays and tissue engineering. To promote cell adhesion, surfaces coated with extracellular matrix (ECM) proteins such as collagen, fibronectin, or their functional sequences (peptides) have been employed. In some cases, polymer-treated surfaces are used in conjunction with the above-mentioned compounds to reduce nonspecific adhesion. Whitesides et al. have reported a variety of cell adhesion patterns, using cell adhesion peptides in combination with ethylene glycol thiolate to prevent nonspecific adsorption of proteins and cells (1-4). In addition, Sabatini et al. reported a cell-based microarray system using cells adhered to gelatin-coated glass slides to identify the cellular functions of gene products (5-7). These methods all utilize the adhesive characteristics of adherent cells. A major limitation of these techniques is that they cannot be applied to nonadherent cells, such as blood cells (especially immunocytes), some cancer cells, and stem cells, all of which are important in various biological and medical fields. As a result, the use of microplates for largescale screenings using nonadherent cells introduces liquid-dispensing difficulties, limits throughput, and significantly adds to the cost. These facts underline the need to develop a cell adhesion method that can be applied generally, such that a miniaturized system without wells can be used in cell-based analysis.

In this study, we introduce a class of cell membrane anchoring reagents, which we will further designate generically as a biocompatible anchor for membrane (BAM). We demonstrate their ability to promote the attachment and to support the growth of both nonadherent and adherent cells. This cell immobilization technique is expected to have application to cell-based microarrays and tissue engineering.

\section{MATERIALS AND METHODS}

\section{General Materials}

Bovine serum albumin (BSA; low endotoxin) was purchased from Sigma (St. Louis, MO, USA), and phosphatebuffered saline (PBS), Dulbecco's modified Eagle medium (DMEM), and RPMI 1640 culture medium were purchased from NISSUI Pharmaceutical Ltd. (Tokyo, Japan). Fetal bovine serum (FBS) was purchased from Asahi Techno Glass (Tokyo, Japan), and cal- cein acetoxymethylester (calcein-AM) was purchased from Molecular Probes (Eugene, OR, USA). The glass-bottomed culture dishes (35-mm uncoated dishes, $1 \mathrm{~cm}$ diameter) were purchased from MatTek (Ashland, MA, USA), the glass slides were purchased from Matsunami Glass Ltd. (Osaka, Japan), and the PD-10 columns were purchased from Amersham Biosciences (Buckinghamshire, UK).

\section{BAM Reagents}

Oleyl-O-poly(ethylene glycol)-succinyl-N-hydroxy-succinimidyl esters [BAM $n$, with $n$ being the average of the ethylene oxide (EO) unit repeats] with three different numbers of $\mathrm{EO}$ units (BAM40, BAM90, and BAM180) in the polyethylene glycol (PEG) moiety and methoxy-poly(ethylene glycol)-succinylN-hydroxy-succinimidyl (NHS) ester with 110 EO unit repeats of PEG (PEGNHS) were gifts from NOF Corporation (Tokyo, Japan). The commercially available equivalents of BAM40, BAM90, BAM180, and PEG-NHS are sold by NOF Corporation with the product names SUNBRIGHT OE-020CS (MW 2000), SUNBRIGHT OE-040CS (MW 4000), SUNBRIGHT OE-060CS (MW 8000 ), and SUNBRIGHT ME-050CS (MW 5000), respectively.

\footnotetext{
${ }^{1}$ National Institute of Advanced Industrial Science and Technology, Hyogo and ${ }^{2}$ University of Tokyo, Tokyo, Japan
} 


\section{Liposomes}

We used freeze-dried liposomes (100- $\mu$ mol lipids/vial) (COATSOME EL Series) of size 100-300 nm, which were gifts from NOF Corporation. Cationic liposome has a dipalmitoyl phosphatidylcholine (DPPC)/cholesterol/stearyl amine ratio of 52:40:8. Nonionic liposome has a DPPC/cholesterol/dipalmitoyl phosphatidylglycerol (DPPG) ratio of 54:40:6. Anionic liposome has a DPPC/cholesterol/ DPPG ratio of 30:40:30.

\section{Cell Lines}

The mouse fibroblast NIH/3T3 cell line was obtained from RIKEN Cell Bank (Tsukuba, Japan). The mouse myeloid cell line 32D was kindly provided by Dr. K. Todokoro (RIKEN, Wako, Japan). K562 human erythroleukemic, Jurkat human $\mathrm{T}$ cell leukemic, and Daudi human Burkitt lymphomic cell lines were obtained from the Institute of Development, Aging, and Cancer, Tohoku University (Sendai, Japan). Mouse embryonic stem (ES) cells and prepared media for the culture of ES cells were purchased from Dainippon Pharmaceutical Ltd. (Osaka, Japan). NIH/3T3 cells were propagated in DMEM supplemented with $10 \%$ FBS, $2.05 \mathrm{mM}$ glutamine, and $30 \mu \mathrm{g} / \mathrm{mL}$ kanamycin at $37^{\circ} \mathrm{C}$ and $5 \% \mathrm{CO}_{2}$. K562, Jurkat, and Daudi cells were cultured in RPMI 1640 medium supplemented with $10 \%$ FBS, $2.05 \mathrm{mM}$ glutamine, and $30 \mu \mathrm{g} / \mathrm{mL}$ kanamycin. 32D cells were grown in RPMI 1640 medium supplemented with the same additives and $2 \mathrm{ng} / \mathrm{mL}$ interleukin-3. Mouse ES cells were cultured in the prepared medium on collagen-coated culture dishes.

\section{Immobilization of Various Suspend- ed Cells on BAM-Modified Surfaces}

The surfaces of glass-bottomed cul- ture dishes were treated aseptically under the following conditions: surfaces were treated with $1 \%$ BSA in PBS at room temperature for $16 \mathrm{~h}$. After washing with Milli- $\mathrm{Q}^{\circledR}$ water (Millipore, Billerica, MA, USA), the surfaces were separately treated with either $100 \mu$ M BAM40, BAM90, BAM180, or PEG-NHS in PBS for $10 \mathrm{~min}$ at room temperature. After washing with Milli-Q water, nonadherent human erythroleukemia K562 cells suspended in the PBS $\left(3 \times 10^{6}\right.$ cells $/ \mathrm{mL}, 100 \mu \mathrm{L}$ in a glass-bottomed culture dish) were transferred onto the BAM-treated surfaces and left for approximately 5 min at room temperature. The surface was then gently washed with PBS to remove cells not immobilized.

Nonadherent cells, human $\mathrm{T}$ cell lymphoma Jurkat, human B cell leukemia Daudi, and mouse myeloid 32D cells were suspended in serum-free RPMI 1640 medium $\left(3 \times 10^{6}\right.$ cells/ $\mathrm{mL}$ ), while adherent mouse ES, hu- 
man embryo kidney (HEK) 293, and mouse fibroblast NIH/3T3 cells were suspended in serum-free DMEM $(3 \times$ $10^{6}$ cells $\left./ \mathrm{mL}\right)$. These cell suspensions were separately transferred onto the BAM90-modified surfaces as described above. 32D, HEK 293, and NIH/3T3 cells were immobilized on the circular BAM90-modified surfaces.

\section{Immobilization of the Liposomes Containing Fluorescein on BAM- Modified Surfaces}

To prepare fluorescein-contained liposomes, $2 \mathrm{~mL}$ of $200 \mu \mathrm{M}$ fluorescein in PBS were added to each vial containing one of the three kinds of empty liposomes (cationic, nonionic, or anionic). To remove free fluorescein, $500 \mu \mathrm{L}$ of suspended reconstituted liposomes were purified separately with PBS by gel-filtration using a PD-10 column. The purified liposomes containing fluorescein were prepared and then resuspended in PBS $(12.5 \mu \mathrm{mol}$

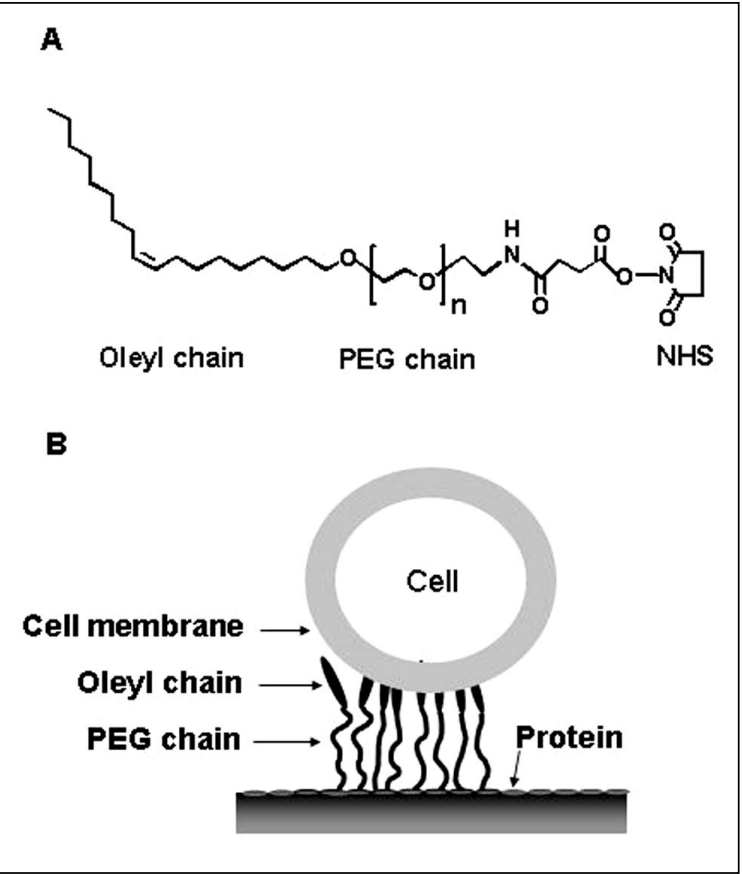

Figure 1. The structure of a biocompatible anchor for membrane (BAM). (A) BAM is composed of an oleyl group and a polyethylene glycol (PEG) chain of various lengths $[n$ is the average number of the ethylene oxide (EO) unit repeats; $n=40,90$, and 180]. Each BAM was modified with N-hydroxysuccinimide (NHS) at the PEG end to react with amino groups. (B) A schematic of cell immobilization shows BAMs reacting with the amino groups of proteins adsorbed on a solid surface. The cells are anchored to the solid surface by interactions between the cell membrane and an oleyl group of BAM (such as insertion of oleyl groups of immobilized BAM into a cell membrane).
lipid/mL). The three liposome suspensions were transferred separately $(100$ $\mu \mathrm{L} /$ glass-bottomed dish) to BAM90or BSA-coated glass-bottomed culture dishes as described above. After 15 min at room temperature, the dishes were gently rinsed with PBS four times to remove nonbinding liposomes. The liposomes were then observed by fluorescence photomi-

\section{Culture of Nonadherent (32D and K562) Cells Immobilized on BAM- Modified Surface}

Approximately $0.2 \mu \mathrm{L}$ of $10 \mu \mathrm{M}$ BAM90 in PBS was spotted onto glass-bottomed culture dishes coated with BSA. The diameter of BAM spots was approximately $1 \mathrm{~mm} 32 \mathrm{D}(2.5 \times$ $10^{5}$ cells $/ \mathrm{mL}, 200 \mu \mathrm{L} /$ glass-bottomed culture dish), and K562 (2.5 × $10^{5}$ ls/mL, $200 \mu \mathrm{L} /$ glass-bottomed dish) were separately immobilized on the BAM-treated glass-bottomed dish. After washing with PBS, $10 \%$ FBS containing RPMI 1640 medium (2 mL) was added to the culture dishes, and the immobilized cells were cultured in a $\mathrm{CO}_{2}$ incubator at $37^{\circ} \mathrm{C}$. During this incubation, the cells were observed using a microscope at 24-h intervals. K562 cells were stained with calcein-AM (at final concentration of $2 \mu \mathrm{M}$ for $15 \mathrm{~min}$ ) to evaluate their viability after 7 days of culture.

\section{Specific Growth Rate of Immobi- lized Cells}

BAM90, BAM180, and PEG-NHS were separately linked to BSA-coated 24-well plates. Three hundred microliters of $1 \times 10^{5}$ cells/mL K562 cells suspended in serum-free RPMI 1640 were added to each treated well. After $10 \mathrm{~min}$, 2 mL RPMI 1640 medium containing $10 \%$ FBS were added to each well. The cells were then cultured for 3 days, and the number of cells was counted at 24-h intervals. Under each condition, three to five experiments were independently performed. The specific growth rates were calculated, and differences in specific growth rates in each of the four conditions were determined by analysis of variance (ANOVA).

\section{Fabrication of High-Density Cell Microarray}

Glass slides were coated with BSA and, after washing with Milli-

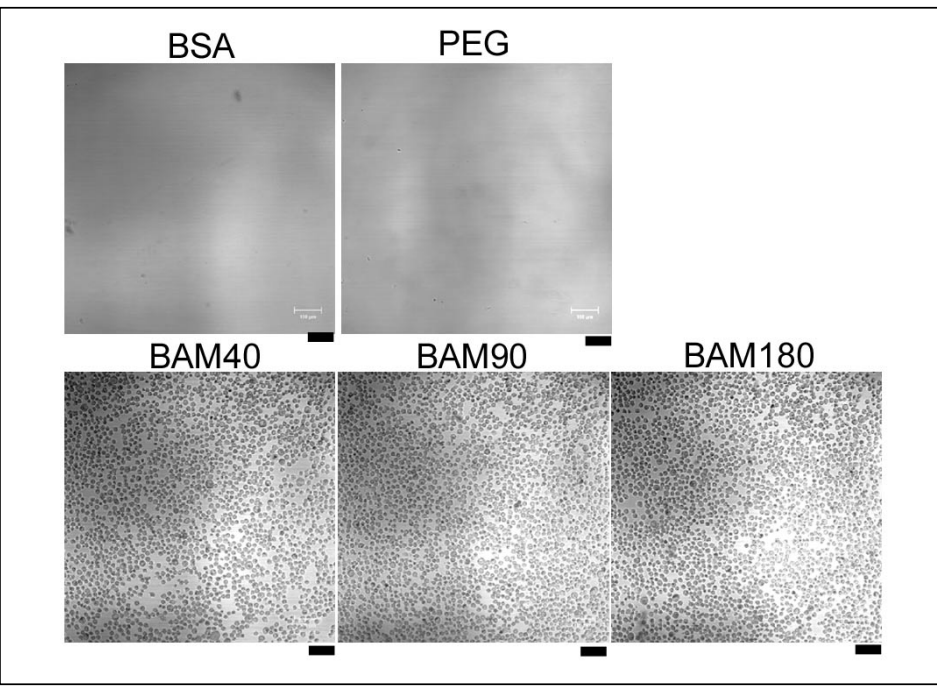

Figure 2. Photomicrographs of immobilized human erythroleukemia K562 cells on the surfaces coated with biocompatible anchors for membranes (BAMs) with polyethylene glycol (PEG) chains of various lengths. The area of the glass-bottomed culture dishes ( $1 \mathrm{~cm}$ diameter) were coated with bovine serum albumin (BSA) and then treated with polyethylene glycol N-hydroxysuccinimide (PEG-NHS), BAM40, BAM90, or BAM180, and also without BAM (i.e., BSA control). K562 cells were added to each prepared dish and then observed by microscopy. All scale bars indicate $100 \mu \mathrm{m}$. 
Q water, were air-dried. Twentynanoliter spots of $10 \mu \mathrm{M}$ BAM90 in aqueous solution were placed onto the glass slide using an ink jet-type printer (Cartesian Technologies, Ann Arbor, MI, USA). The BAM spot was approximately $500 \mu \mathrm{m}$ in diameter. Likewise, 432 BAM spots were placed in an array onto the glass slide. The pattern was constructed with $4 \times 12$ subarrays, where each subarray contained $3 \times 3$ spots (432 spots total). After a few minutes, the BAM microprinted glass slide was rinsed with Milli-Q water. The BAMmodified glass slide was then dried and stored at room temperature until further use. To prepare a high-density cell microarray, $2 \mathrm{~mL}$ of the $32 \mathrm{D}$ cell suspension $\left(1 \times 10^{6}\right.$ cells $\left./ \mathrm{mL}\right)$ in serum-free RPMI 1640 were added to the surface of the BAM-arrayed glass slide. After $10 \mathrm{~min}$, the glass slide treated with cells was gently rinsed with PBS to remove cells not immobilized on the BAM spots. The cells, which were placed in dense arrays on the glass slide, were fixed with $100 \%$ ethanol for image scanning. The back of cell-microarrayed glass slide was covered with black paper to permit clear visualization of the cell array. The image of the glass slide was obtained using an image scanner, and one of the cell clusters was observed by microscopy.

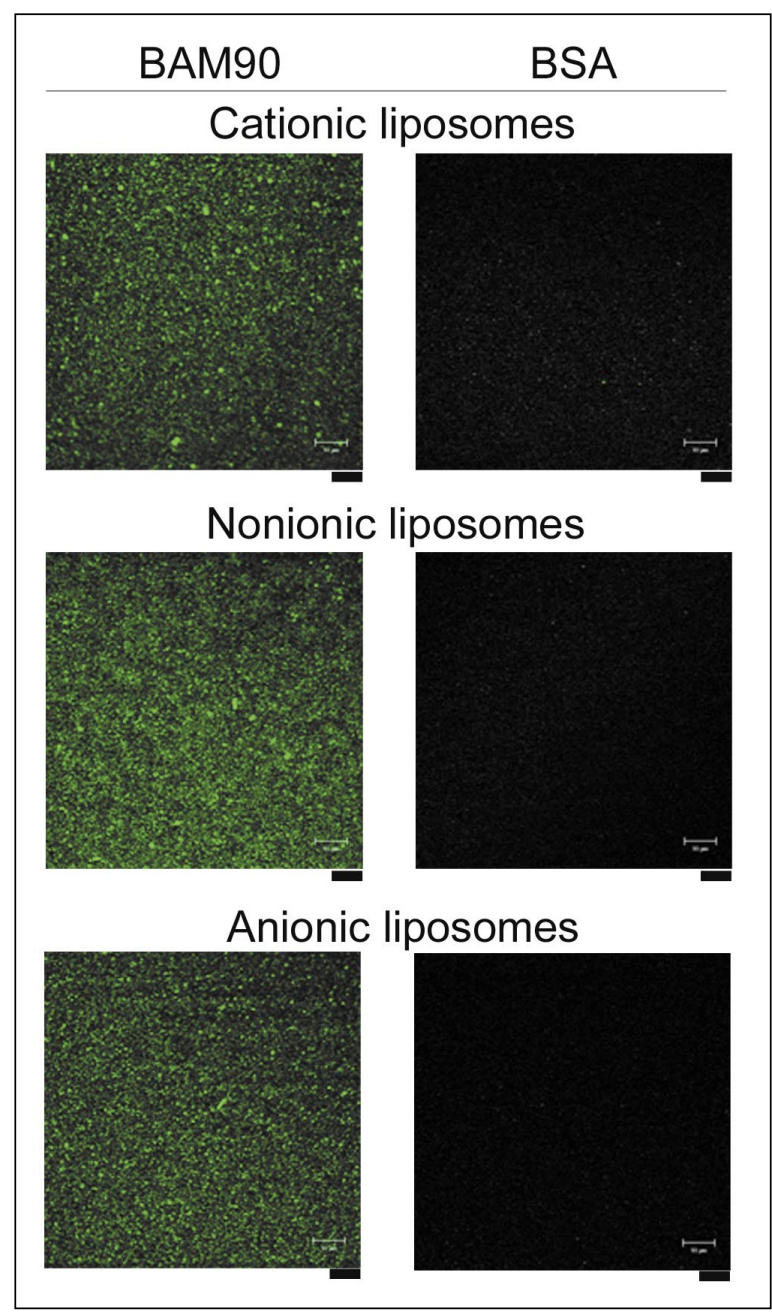

Figure 3. Photomicrographs of various liposomes containing fluorescein on the biocompatible anchor for membrane (BAM)or bovine serum albumin (BSA)-modified surfaces. Cationic, nonionic, and anionic liposomes encapsulating fluorescein were added to each prepared surface, and then after washing with phosphatebuffered saline (PBS), the immobilized liposomes were observed by fluorescence microscopy. All scale bars indicate $100 \mu \mathrm{m}$.

\section{RESULTS AND DISCUSSION}

Immobilization of Cells on BAMModified Surfaces

BAM consists of three distinct units: $(i)$ a hydrophobic oleyl group; (ii) a hydrophilic PEG of variable length; and (iii) an amino-reactive NHS group (Figure 1A). We prepared a series of BAM (BAM40, BAM90, and BAM180)-modified surfaces in glass-bottomed culture dishes precoated with BSA and added suspended human erythoroleukemia K562 cells to the surface. The cells were immobilized on the BAM-modified surfaces, but not on surfaces covered with PEG or BSA (Figure 2). These results suggest that an oleyl chain is an important lipid anchor that can bind cells to the BAM-modified surface. Moreover, we found that precoating the surface with ovalbumin, human serum albumin, human $\gamma$-globulin, or $\alpha$-casein instead of BSA was equally successful, since the surface of these proteins possesses a sufficient number of amino groups for BAM conjugation on the surface (data not shown). Additionally, liposomes that were only composed of lipids were also immobilized on BAM-modified surfaces. This binding was not dependent on the electrostatic charge of the liposomes (Figure 3), implying that the oleyl group of BAM is targeted to lipid bilayers.

The binding of cells to the BAM surface was also achieved in a PBS(-) solution (i.e., PBS lacking metal ions). Since a simple lipid bilayer (as in each of these three kinds of liposomes) could

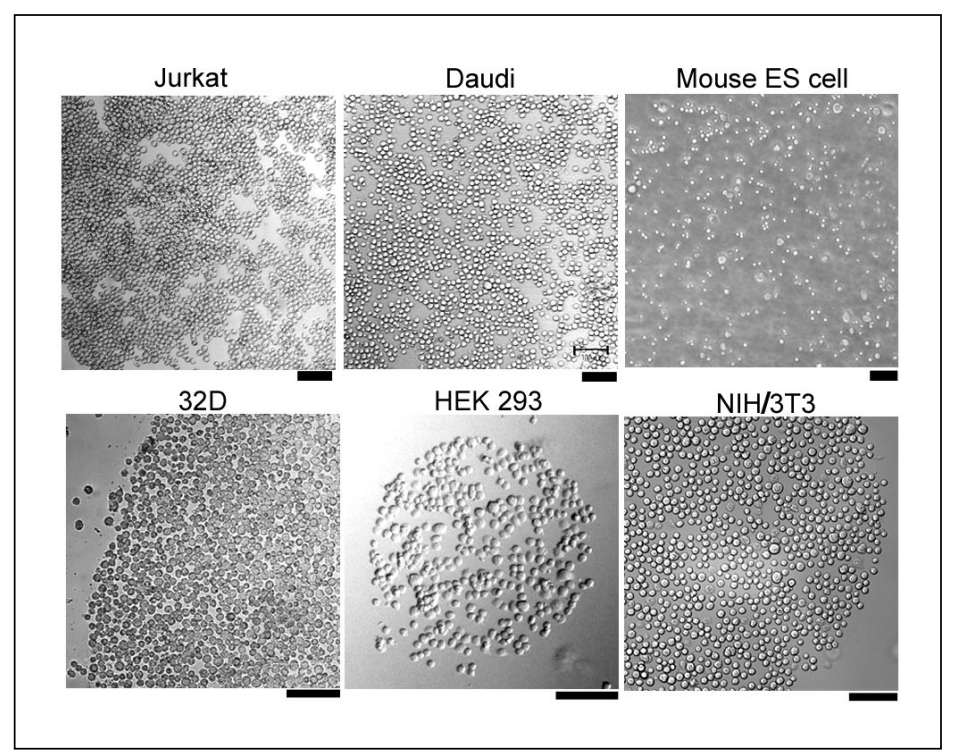

Figure 4. Photomicrographs of various cells immobilized on a biocompatible anchor for membrane (BAM)90-coated glass surfaces. Human T cell lymphoma Jurkat, human B cell leukemia Daudi, mouse embryo stem (ES), and mouse myeloid 32D cells were separately immobilized on BAM90-coated surfaces. The suspended adherent cells, human embryo kidney (HEK) 293 and mouse fibroblast NIH/3T3, were quickly immobilized on the circular BAM90-modifed surface. All scale bars indicate $100 \mu \mathrm{m}$. 

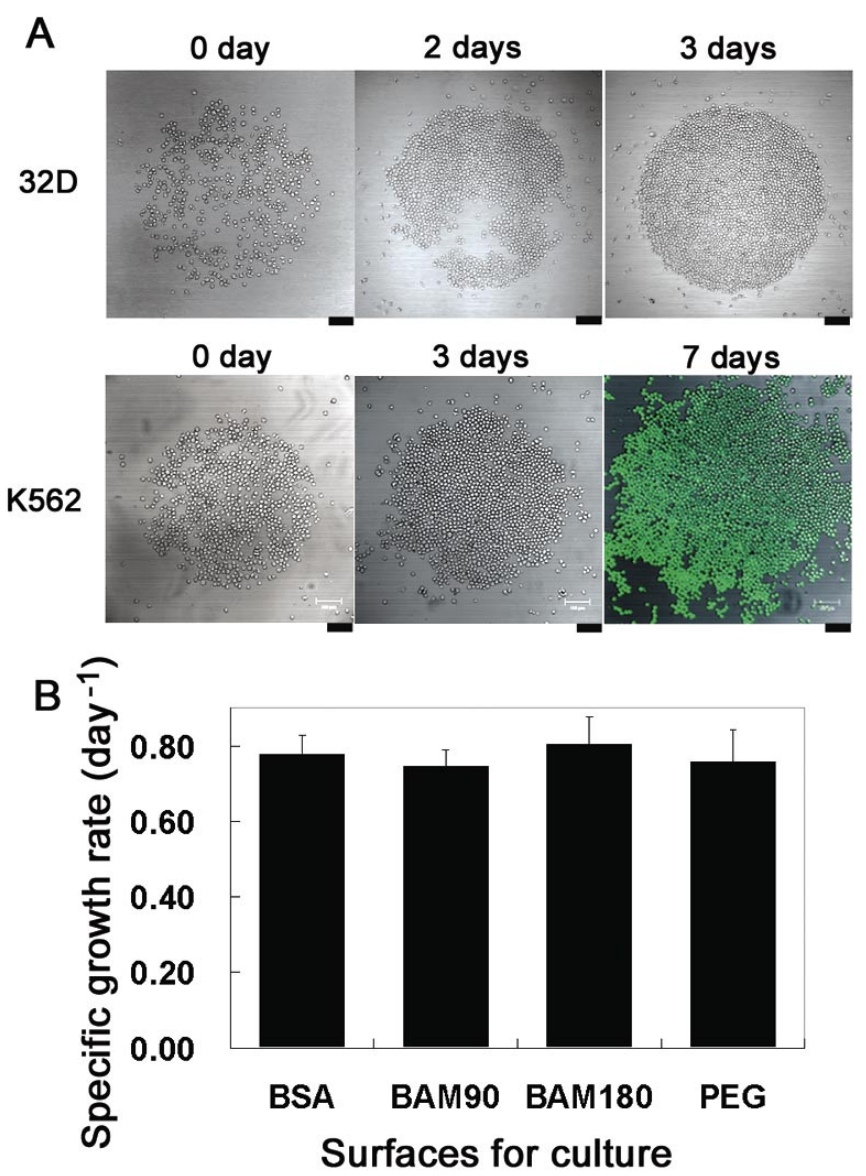

Figure 5. Growth of cells immobilized on the surface of a biocompatible anchor for membrane (BAM). (A) Photomicrographs of 32D and K562 cells cultured for the number of days indicated. Cells were grown in RPMI 1640 medium containing 10\% fetal bovine serum (FBS) with immobilization on BAM90-treated glass surfaces ( $1 \mathrm{~mm}$ diameter). Fluorescence image of $\mathrm{K} 562$ cells were stained with calcein acetoxymethylester (calcein-AM) after 7 days to determine cell viability. All scale bars indicate $100 \mu \mathrm{m}$. (B) Comparison of cell growth rates on BAM-modified surfaces over 3 days.
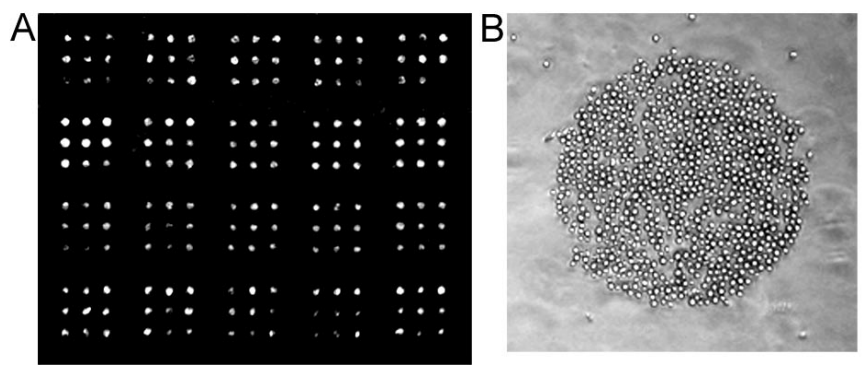

Figure 6. Fabrication of a high-density cell array using an ink jet printer. (A) Image scanning photo of arrayed 32D cell clusters on glass slides with the back covered by black paper. Photo dimensions are $19 \mathrm{~mm}$ high and $23.6 \mathrm{~mm}$ wide. The biocompatible anchor for membrane (BAM) solution was arrayed with a pattern consisting of $4 \times 12$ blocks containing $3 \times 3$ spots $(432$ spots total) on the bovine serum albumin (BSA)-coated glass slides using an ink jet printer. (B) Photomicrographs of K562 cells immobilized on a BAM-coated spot. The diameter of spot was approximately $500 \mu \mathrm{m}$. also be immobilized on the BAM surface irrespective of the difference in surface charge (Figure 3), cell immobilization via a lipid anchor probably does not require an interaction between lipid anchors and proteins or sugar chains on the cell membrane. From these results, it may be concluded that cells are immobilized on BAM surfaces via interactions between the oleyl chain of BAM and the cell membrane (i.e., insertion of oleyl chain into cell membrane) without requiring mediators such as proteins, sugar chains, and metal ions.

In light of these results, we further studied the adhesion profiles of other nonadherent cells on BAM90-modified surfaces. Human T cell lymphoma Jurkat, human B cell leukemia Daudi, mouse ES, and mouse myeloid 32D cells all successfully adhered to the BAM90 surface (Figure 4). We also tested our protocol on the immobilization of adherent cells. HEK 293 and mouse fibroblast NIH/3T3 cells were rapidly immobilized on a BAM90 surface within $5 \mathrm{~min}$ (Figure 4). This result indicates that immobilization using BAM is faster than the natural binding of adherent cells via adhesive proteins, such as integrin, on the cell membrane and strongly supports an alternative mechanism of adhesion. However immobilized adherent cells could not survive on these surfaces even for 1 day. This is probably attributable to these cells being dependent on anchoring and hence could not grow in the absence of cellular adhesion to ECM proteins. When collagen replaced BSA as a coating protein, NIH/3T3 cells adhered and grew on the BAM-modified surface of collagen. However, NIH/3T3 cell growth on this surface was slightly inferior to that observed on collagen surfaces. These results indicate that the immobilization mechanism of adherent cells by BAM is different to that of integrin-mediated cell adhesion.

\section{Culture of Immobilized Cells and Comparison of Growth Rate}

For cell-based analysis, a cultivation surface must support long-term cell survival in addition to its cell adhesion properties. This feature was examined in mouse myeloid 32D and human erythroleukemia K562 cells, which were im- 
mobilized on BAM90 surfaces. The immobilized cells were cultivated in RPMI 1640 medium containing 10\% FBS. After 3 days, both cell types proliferated confluently in the BAM90 area (Figure $5 \mathrm{~A})$. These results clearly indicate that immobilized cells proliferate on the BAM surface.

The culture of immobilized K562 was continued for a total of 7 days, and their viability was then confirmed by a calcein-AM stain (Figure 5A). These immobilized cells did not detach in RPMI 1640 medium with 10\% FBS, and interestingly, the cell clusters were slightly larger than the circular BAM area. This is probably due to cell-cell or cell-surface interactions specific for K562 cells, since this phenomenon was not observed in 32D cells.

Furthermore, we studied whether the specific growth rates of cells are affected by the nature of the BAM-conjugated surface. K562 cells were cultured on each surface coated with BSA, BAM90, BAM180, or PEG in a 24-well microplate. The cells on the surface coated with BSA or PEG were not immobilized. In contrast, the cells cultured on the surfaces coated with BAM90 or BAM180 were immobilized. The number of cells under each condition was counted every $24 \mathrm{~h}$ over a 3 -day period. Our results show that there was no significant difference $(P>0.05$, by ANOVA) between the four specific growth rates (Figure 5B). We conclude that the immobilization of cells on a BAM surface does not influence cell growth.

\section{Fabrication of High-Density Cell Microarray}

Based on the above studies, we fabricated a high-density cell microarray using BAM90. The BAM90 spots were microprinted onto a BSA-coated glass slide, as described in the Materials and Methods section. After the reaction and subsequent washing, the addition of the cell suspension onto the BAM-spotted glass slides resulted in their immediate immobilization (Figure 6A). Each of the cell clusters on the BAM spots included approximately 1000 confluent living cells (Figure 6B).

In conclusion, use of the newly developed BAM series of reagents has enabled us to prepare novel functional surfaces, which can be utilized in new cell culture techniques using the interactions between the cell membrane and the oleyl group of BAM. Considering that up until now, the cell microarrays have been limited to adherent cells, our technique significantly extends the versatility to include nonadherent cells, such as immunocytes, stem cells, and cancer cells lacking anchorage-dependency.

\section{ACKNOWLEDGMENTS}

We thank T. Yasukouchi, C. Ito, S. Ohhashi, K. Kubo, and other members of NOF Corporation for the synthesis of BAMs and PEG-NHS.

\section{REFERENCES}

1.Zhang, S., L. Yan, M. Altman, M. Lassle, H. Nugent, F. Frankel, D.A. Lauffenburger, G.M. Whitesides, and A. Rich. 1999. Biological surface engineering: a simple system for cell pattern formation. Biomaterials 20: 1213-1220.

2.Chen, C.S., M. Mrksich, S. Huang, G.M. Whitesides, and D.E. Ingber. 1997. Geometric control of cell life and death. Science 276 : 1425-1428.

3.Chen, C.S., M. Mrksich, S. Huang, G.M. Whitesides, and D.E. Ingber. 1998. Micropatterned surfaces for control of cell shape, position, and function. Biotechnol. Prog. 14: 356-363.

4.Ostuni, E., L. Yan, and G.M. Whitesides. 1999. The interaction of proteins and cells with self-assembled monolayers of alkanethiolates on gold and silver. Colloids Surf. B Biointerfaces 15:3-30.

5.Ziauddin, J. and D.M. Sabatini. 2001. Microarrays of cells expressing defined cDNAs. Nature 411:107-110.

6.Wu, R.Z., S.N. Bailey, and D.M. Sabatini. 2002. Cell-biological applications of transfected-cell microarrays. Trends Cell Biol. 12: 485-488.

7.Bailey, S.N., R.Z. Wu, and D.M. Sabatini. 2002. Applications of transfected cell microarrays in high-throughput drug discovery. Drug Discov. Today 7:S113-S118.

Received 4 July 2003; accepted 26 August 2003.

Address correspondence to:

Teruyuki Nagamune

Department of Chemistry and Biotechnology

School of Engineering

The University of Tokyo

7-3-1 Hongo

Bunkyo-ku, Tokyo 113-8656, Japan

e-mail:nagamune@bio.t.u-tokyo.ac.jp 